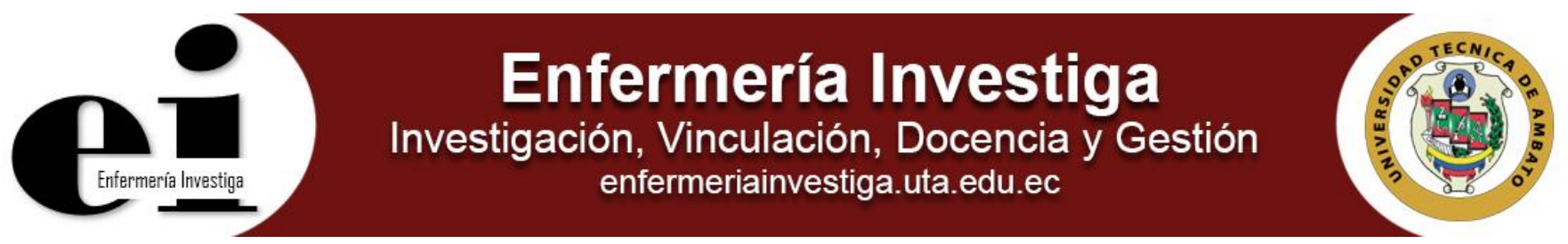

DOI: $\underline{\text { http://dx.doi.org/10.29033/enfi.v4i1.475 }}$

Artículo original

\title{
Síntomas iniciales en niños con maltrato infantil
}

Initial symptoms in children with child maltreatment

María José Terán Bejarano ${ }^{1}$, Carlos Antonio Escobar Súarez², Anabel Cristina Terán Bejarano³, Baiter Renán Cazares Cadena ${ }^{4}$, Paulina Elizabeth Durán Mora ${ }^{5}$, Giovanna Paulina Posso López ${ }^{6}$, Patrick Alexander Durán Acosta7 ${ }^{7}$, Mónica Elizabeth Tovar Gavilanes ${ }^{8}$, Ana Lucía Palacios Sánchez ${ }^{9}$

\footnotetext{
${ }^{1}$ Hospital General Docente Ambato - Servicio Emergencia - Sala de Primera Acogida - Ambato - Ecuador

${ }^{2}$ Universidad Central del Ecuador - Carrera de Atención Prehospitalaria y Emergencia - Ambato-Ecuador

${ }^{3}$ Centro Médico Popular - Servicio de Odontología - Ambato - Ecuador

${ }^{4}$ Universidad Central del Ecuador - Carrera de Atención Prehospitalaria y Emergencia - Quito - Ecuador

${ }^{5}$ Hospital General Durán - Servicio de Cirugía - Ambato - Ecuador

${ }^{6}$ Centro de Salud Loma Grande Tipo A - Latacunga - Ecuador

${ }^{7}$ Hospital General Docente Ambato - Servicio de Cirugía - Ambato - Ecuador

${ }^{8}$ Hospital General Ibarra - Servicio de Anestesiología - Ibarra - Ecuador

${ }^{9}$ Unidad Asistencial San Pablo de Ushpsyacu - Centro de Salud Tipo A - Archidona - Ecuador
}

Terán MJ, Escobar CA, Terán AC, Cazares BR, Durán PE, Posso GP, Durán PA, Tovar ME, Palacios AL. Síntomas iniciales en niños con maltrato infantil. Enferm Inv. 2019; 4(1):2-7

2477-9172 / 2550-6692 Derechos Reservados ( 2019 Universidad Técnica de Ambato, Carrera de Enfermería. Este es un artículo de acceso abierto distribuido bajo los términos de la Licencia Creative Commons, que permite uso ilimitado, distribución y reproducción en cualquier medio, siempre que la obra original es debidamente citada.

\section{Historia:}

Recibido: 15 octubre 2018

Revisado: 14 noviembre 2018

Aceptado: 18 marzo 2019

Palabras Claves: Maltrato infantil, violencia

Keywords: Child abuse, violence

\begin{abstract}
Resumen
Introducción: Alrededor de 300 millones de niños y niñas en el mundo viven en situación de violencia, 70 millones de ellos en Latinoamérica, constituye uno de los problemas principales de salud pública en todo el mundo.
\end{abstract}

Objetivo: Identificar los síntomas iniciales de niños con maltrato infantil atendidos en Sala de Primera Acogida del Hospital General Docente Ambato en el año 2017, para la obtención de un diagnóstico presuntivo.

Métodos: Estudio retrospectivo, observacional, descriptivo, a través de entrevistas a los médicos que atendieron los niños maltratados y se realizó una observación científica. En una población de 437 niños atendidos en el año 2017, teniendo como muestra de estudio 50 pacientes elegidos por criterios de inclusión y exclusión.

Resultados: Se obtuvo como resultado síntomas conductuales (95\%), miedo (75\%), depresión (65\%), trastornos del sueño y alimentación (45\%), como síntomas principales de un niño maltratado. Se elaboró una guía de detección y diagnóstico que facilite al personal de salud un diagnóstico temprano de maltrato infantil.

Conclusiones: Los síntomas iniciales en niños maltratados demostraron afectación en el desenvolvimiento psicológico y físico normal del paciente menor de edad, en la que influyen varios factores del entorno social, familiar y los situacionales, en especial las variables cognitivas de las víctima que han permitido explicar la gravedad de la sintomatología, de la cual requiere un tratamiento, seguimiento adecuado y oportuno en un lapso de tiempo ideal.

\section{Abstract}

Introduction: Around 300 million children in the world live in a situation of violence, 70 million of them in Latin America, which constitutes one of the main problems of public health in the whole world

Objective: To identify the initial symptoms of children with child abuse treated in the First Reception Room of the Ambato General Teaching Hospital in 2017, in order to obtain a presumptive diagnosis.

Methods: A retrospective study, observational, and descriptive, through interviews with the doctors who treated the abused children. In a population of 437 children attended in the year 2017, having as sample of study 50 patients chosen by inclusion and exclusion criteria.

Results: Behavioral symptoms (95\%), fear (75\%), depression $(65 \%)$, sleep disorders and feeding $(45 \%)$ were the main symptoms of a battered child. A screening and diagnosis guide was developed to provide health personnel with an early diagnosis of child maltreatment.

Conclusions: The initial symptoms in abused children showed affectation in the normal psychological and physical development of the minor patient, in which several factors of the social, familiar and situational environment influence, especially the cognitive variables of the victims that

have allowed to explain the severity of the symptomatology, which requires treatment, adequate and timely follow-up in an ideal period of time.

Autor de correspondencia:

María José Terán Bejarano. Hospital General Docente Ambato - Servicio Emergencia - Sala de Primera Acogida. Teléfono: +593 983426110, Ambato, Ecuador. Email: majosteran@hotmail.com 


\section{Introducción}

El maltrato infantil afecta el desarrollo físico y mental de los niños que lo sufren. Con el transcurso del tiempo esta situación se incrementa. Se puede manifestar de varias formas: maltrato físico, maltrato psicológico, abuso sexual, explotación, negligencias o abandono.

En los datos del último informe de UNICEF, se evidencia que una quinta parte de las víctimas de homicidio en todo el mundo son niños y adolescentes menores de 20 años, siendo la región de América Latina y el Caribe la que tiene la proporción más alta de víctimas de homicidio de menores de 25 años (25.400). A nivel mundial, otro dato alarmante es que alrededor de 120 millones de mujeres menores de 20 años (1 de cada 10) han experimentado relaciones sexuales por la fuerza u otros actos sexuales forzados..$^{1,2}$

De cualquier modo, los estudios internacionales de la Organización Mundial de la Salud (OMS), revelan que aproximadamente un $20 \%$ de las mujeres y un $5 \%$ a $10 \%$ de los hombres manifiestan haber sufrido abusos sexuales en la infancia, mientras que un $25 \%$ a $50 \%$ de los niños de ambos sexos refieren maltratos físicos. Además, muchos niños son objeto de maltrato psicológico y víctimas de desatención..$^{3,4}$

En Ecuador en el año 2011, 204 personas de 10 a 19 años murieron por homicidio y 223 por suicidio (8.6\% y $9.4 \%$ del total de muertes en este grupo etario respectivamente), según datos de las Estadísticas Vitales 2011. Y de acuerdo a la Encuesta Nacional de Violencia de Género y la Agenda Nacional de las Mujeres e Igualdad de Género (2014-2017), 6 de cada 10 mujeres han sufrido algún tipo de violencia: psicológica, física, sexual y/o patrimonial; y 1 de cada 10 ha sufrido abuso sexual antes de cumplir los 18 años. El porcentaje de los niños con maltrato infantil de tipo fisco se mantiene prácticamente en los mismos niveles registrados en los años 2010 y 2011 , es decir alrededor del $40 \%$ en el Ecuador. ${ }^{3}$

En lo que corresponde al trato que reciben los niños por parte de los profesores en sus escuelas o instituciones educativas, asimismo ante faltas o no cumplimiento de deberes, el mencionado documento indica que el $10 \%$ de los niños reciben golpes y el 7\% insultos y burlas. (4) Además, informa que otras formas de castigo son: no permitirles salir al recreo, rebajar las calificaciones, llamar a los representantes. Aproximadamente, el $26 \%$ de los niños dicen que los profesores sí dialogan con ellos. Como una de las conclusiones, se dice que hay indiferencia de los profesores en un 12\%. El maltrato a la niñez y adolescencia en el Ecuador es alarmante, tomando en cuenta que éste no se refiere únicamente al castigo físico, sino a cualquier acción u omisión que afecta el normal desenvolvimiento de los menores. ${ }^{5}$

Según el Observatorio de los Derechos de la Niñez y la Adolescencia en el Ecuador, el número de menores que vive en extrema pobreza se redujo al $18 \%$ en 2010 y también bajó el trabajo infantil, pero al mismo tiempo se elevó al $42 \%$ el porcentaje de niños que sufre maltrato y el embarazo adolescente se duplicó. ${ }^{4}$

Las formas más comunes de maltrato contra este sector de la población son el psicológico y físico, aunque se registran también datos por violencia sexual y maltrato institucional. Las estadísticas del Instituto Nacional de la Niñez y la Familia (INFA) demuestran que en las ciudades de Quito y Guayaquil el 27\% de la población hasta 10 años es castigada físicamente al menos una vez por semana. En la provincia del Guayas según cifras del INFA obtenidas en los centros de restitución de derechos que funcionan en Guayas, en el 2010 se reportaron 2.650 casos de abandono o negligencia de los padres o responsables del cuidado de los menores de edad. Ese es el principal tipo de maltrato; le siguen las agresiones físicas, con 980 hechos; psicológicas con 837 denuncias; y abuso sexual que dejó en un año 850 víctimas. ${ }^{5}$

Según datos del Instituto Nacional de Estadísticas y Censos (INEC), en el país la violencia, el maltrato, la negligencia y el abuso, constituyen experiencias cotidianas para 7 de cada 10 niños, niñas y adolescentes, en sus diferentes entornos: familiar, escolar, comunitario y social. Constituyendo el $51 \%$ a maltrato psicológico, el $49 \%$ a físico, en total de 987 denuncias de maltrato infantil registrado por la Dirección Nacional de Policía Especializada para Niños, Niñas y Adolescentes (DINAPEN) el año 2012. Esa cifra colocó a Pichincha como la provincia donde más agresiones a menores se registran, 421 abusos sexuales, 407 abusos físicos, y 155 psicológicos, ubicándola en el primer lugar, seguida por Manabí y el Oro. ${ }^{4}$

El Concejo Cantonal de la Niñez y Adolescencia de Tungurahua manifiesta que generalmente el $80 \%$ de maltrato infantil proviene por parte de la madre, $10 \%$ del padre y otro $10 \%$ de sus cuidadores y maestros ya sea maltrato físico, verbal, psicológico o sexual. Las estadísticas en la provincia de Tungurahua realmente son alarmantes su incremento en el 2017 fue aproximadamente del $30 \%{ }^{6}$

La presente investigación es pertinente a esta proyección del Estado ecuatoriano, al enfocarse en la identificación de los síntomas iniciales de niños con maltrato infantil atendidos en Sala de Primera Acogida del Hospital General Docente Ambato en el año 2017, para la obtención de un diagnóstico presuntivo. La importancia del desarrollo de este tema es poder identificar los síntomas iniciales en un niño maltratado y a su vez capacitar a los médicos en el diagnóstico inmediato, ya que muchos pacientes son ingresados y tratados con diagnósticos secundarios a un maltrato infantil. ${ }^{7}$ Investigar sobre el problema es de vital importancia para determinar las causas que impulsan a que los menores sean maltratados por personas dentro del mismo círculo familiar o externo a este y además que corran el riesgo de convertirse en maltratadores. ${ }^{8}$ 
De acuerdo con la Secretaria Nacional de Planificación y Desarrollo, Ecuador estará enfocado en el período 2017 2021 en garantizar el derecho a la salud de sus ciudadanos, apoyado en la promoción de prácticas saludables de vida, encauzadas a la prevención de enfermedades. ${ }^{1}$

Por lo cual, la Sala de Primera Acogida (SPA) tiene el papel fundamental al permitir el abordaje precoz que favorece una evaluación de la situación. Y el diagnóstico de los posibles daños. Existen varios indicadores para considerar en la problemática: alimentación inadecuada, carencias de vestimenta, higiene, cuidados médicos, falta de supervisión adulta, condiciones peligrosas en el entorno domiciliario o problemas escolares.

\section{Objetivo General}

Identificar los síntomas iniciales de niños con maltrato infantil atendidos en Sala de Primera Acogida del Hospital General Docente Ambato en el año 2017, para la obtención de un diagnóstico presuntivo.

\section{Métodos}

Los ovarios son glándulas femeninas, que producen óvulos para la reproducción, además hormonas como estrógenos y progesterona. Los ovarios tienen tres tipos de tejidos: Células epiteliales que cubren el ovario. Células

La investigación fue de tipo retrospectivo, observacional, descriptiva. Se realizaron entrevistas a los médicos que atendieron a pacientes objeto del estudio, se utilizó una ficha de recolección de datos de pacientes hospitalizados y se realizó una observación científica. En una población de 437 niños atendidos en el año 2017, teniendo como muestra de estudio 50 pacientes elegidos por criterios de inclusión y exclusión.

\section{Criterio de Inclusión:}

- Pacientes ingresados al servicio de Pediatría

- Pacientes que permitan bajo autorización el seguimiento observacional de comportamiento y a sus padres

- Pacientes que tengan aperturada la historia clínica

\section{Criterios de Exclusión:}

- Pacientes que fueron atendidos una sola vez como consulta externa

- Pacientes que padres no permitan con autorización la realización del seguimiento Pacientes que no nos proporcionen la información adecuada y completa.

\section{Procedimientos y técnicas}

La recolección de la información se cumplió con los respectivos permisos autorizados por el área de investigación y docencia del Hospital General Docente Ambato, que facilitó el acceso a los datos estadísticos por el departamento de admisiones a las historias clínicas de los pacientes atendidos por la Sala de Primera Acogida del Hospital General Docente Ambato en el año 2017.

La ficha de recolección de datos, posibilita desarrollar la técnica de documentación, mediante la revisión de las historias clínicas de cada uno de los pacientes atendidos. Los datos obtenidos se basaron en los aspectos sociodemográficos, conductuales, tipo de maltrato y periodicidad.

\section{Resultados}

Tabla 1: Niños atendidos en Sala de Primera Acogida del Hospital General Docente Ambato

\begin{tabular}{|l|l|l|l|l|l|}
\hline Grupos de edad & Hombre & $\%$ & Mujer & $\%$ & Total atenciones \\
\hline 1-11 MESES & 11 & $50 \%$ & 11 & $50 \%$ & 22 \\
\hline 1-4 AÑOS & 42 & $51.85 \%$ & 39 & $48.15 \%$ & 81 \\
\hline 5-9 AÑOS & 65 & $55.08 \%$ & 53 & $44.92 \%$ & 118 \\
\hline 10-14 AÑOS & 41 & $32.54 \%$ & 85 & 67.46 & 126 \\
\hline TOTAL & 159 & $45.82 \%$ & 188 & $54.18 \%$ & $\mathbf{3 4 7}$ \\
\hline
\end{tabular}

\section{Análisis e Interpretación:}

En el estudio realizado se atendió un total de 347 niños que presentaron indicadores de maltratado en su primera consulta demostrándose mayor incidencia en el sexo femenino y mayor predominio en la edad comprendida entre los 10 a 14 años.

Tabla 2: Sintomatología inicial en niños maltratados físicamente 


\begin{tabular}{|l|l|l|}
\hline Síntomas en maltrato físico & $\mathrm{n}$ & $\%$ \\
\hline Miedo & 87 & 25,07 \\
\hline Enuresis & 37 & 10,66 \\
\hline Bajo rendimiento escolar & 25 & 7,20 \\
\hline Insomnio & 25 & 7,20 \\
\hline Cambios conductuales & 80 & 23,05 \\
\hline Malestar Físico & 43 & 12,39 \\
\hline Depresión & 25 & 7,20 \\
\hline Aislamiento social & 25 & 7,20 \\
\hline Total & 347 & 100,00 \\
\hline Fuente: Ficha de recolección de datos & &
\end{tabular}

\section{Análisis e Interpretación:}

La sintomatología por maltrato físico en niños ocasiona miedo por parte del menor hacia sus padres o cualquier otra persona que cumpla con el perfil del agresor, demostrando secundariamente cambios conductuales marcados como por ejemplo la agresividad, el aislamiento social, la rebeldía, la depresión, etc.

Tabla 3: Sintomatología inicial en niños maltratados sexualmente

\begin{tabular}{|l|l|l|l|l|l|l|}
\hline \multirow{2}{*}{$\begin{array}{l}\text { Síntomas por maltrato } \\
\text { sexual }\end{array}$} & \multicolumn{5}{|l|}{ a 9 años } & \multicolumn{2}{l|}{$\mathbf{1 0}$ a 14 años } & \multicolumn{2}{l|}{ Total } \\
\cline { 2 - 8 } & $\mathbf{n}$ & $\%$ & $\mathbf{n}$ & $\%$ & $\mathbf{n}$ & $\%$ \\
\hline Síntomas conductuales & 80 & 26,94 & 22 & 44 & 102 & 29,39 \\
\hline Trastornos del sueño & 25 & 8,42 & 5 & 10 & 30 & 8,65 \\
\hline Enuresis & 37 & 12,46 & 2 & 4 & 39 & 11,24 \\
\hline Miedo & 87 & 29,29 & 7 & 14 & 94 & 27,09 \\
\hline Bajo rendimiento escolar & 25 & 8,42 & 9 & 18 & 34 & 9,80 \\
\hline Malestar Físico & 43 & 14,48 & 5 & 10 & 48 & 13,83 \\
\hline Total & 297 & 100,00 & 50 & 100 & 347 & 100,00 \\
\hline Fuente: Ficha de recolección de datos & & & & & &
\end{tabular}

\section{Análisis e Interpretación:}

La sintomatología por maltrato sexual da mayor incidencia al miedo en pacientes comprendidos entre los 5 a 9 años de edad, seguidos de síntomas típicamente conductuales, mientras que en los pacientes de 10 a 14 años de edad hay una incidencia marcada en los síntomas conductuales, es decir que los niños se ven afectados psicológicamente convirtiéndose este en un trastorno postraumático y con consecuencias aún más fuertes en el desarrollo del paciente menor de edad.

Tabla 4: Sintomatología inicial en niños maltratados psicológicamente

\begin{tabular}{|l|l|l|l|l|l|l|}
\hline \multirow{2}{*}{$\begin{array}{l}\text { Síntomas por maltrato } \\
\text { psicológico }\end{array}$} & \multicolumn{5}{|l}{$\mathbf{5}$ a 9 años } & \multicolumn{2}{l|}{$\mathbf{1 0}$ a 14 años } & \multicolumn{2}{l|}{ Total } \\
\cline { 2 - 8 } & $\mathrm{n}$ & $\%$ & $\mathrm{n}$ & $\%$ & $\mathrm{n}$ & $\%$ \\
\hline Ansiedad & 25 & 8,42 & 15 & 30,00 & 40 & 11,53 \\
\hline Timidez & 105 & 35,35 & 2 & 4,00 & 107 & 30,84 \\
\hline Depresión & 4 & 1,35 & 4 & 8,00 & 8 & 2,31 \\
\hline Baja autoestima & 4 & 1,35 & 5 & 10,00 & 9 & 2,59 \\
\hline Trastornos del sueño & 101 & 34,01 & 2 & 4,00 & 103 & 29,68 \\
\hline Trastornos alimenticios & 56 & 18,86 & 4 & 8,00 & 60 & 17,29 \\
\hline
\end{tabular}




\begin{tabular}{|l|l|l|l|l|l|l|} 
Lesiones autoinflingídas & 2 & 0,67 & 18 & 36,00 & 20 & 5,76 \\
\hline Total & 297 & 100,00 & 50 & 100,00 & 347 & 100,00 \\
\hline Fuente: Ficha de recolección de datos
\end{tabular}

\section{Análisis e Interpretación:}

La sintomatología por maltrato psicológico se refleja en la incidencia en la edad de 5 a 9 años de timidez, al momento de poder exponer que es lo que le está sucediendo que puede ser por miedo, amenazas, etc., por lo cual se ven reflejadas en los trastornos del sueño con pesadillas y miedos nocturnos, mientras que a los pacientes de 10 a 14 años estos síntomas conllevan a situaciones de afectación física directa lo que se conoce como cutting, el hecho descrito en el silencio de auto lastimarse tratando de marcar ese evento en su desarrollo social cotidiano, por lo cual es importante al captar este tipo de pacientes darle el seguimiento adecuado.

\section{Discusión}

Al hablar de maltrato infantil se relacionan diferentes conceptualizaciones y paradigmas que aún se viven en algunos países y se consideran normales, pero debido a la gran incidencia de agresión es que ha llamado la atención poder reflejar la realidad nacional y buscar métodos, técnicas adecuadas para evitar tanto la presencia de este mal social, como la disminución a tiempo de consecuencias de gran importancia social sobre todo el entorno familiar.

Por otra parte, el autor Ramírez Q y Ricardo R, en el 2018, realizan un estudio en la que comprueban que el maltrato infantil es un problema universal y multicausal que involucra una gran variedad de factores biopsicosociales. Es toda agresión producida a los menores por sus padres, familiares u otras personas para castigarles o hacerles daño, incluida también la desatención de sus necesidades básicas de alimentación, hogar, vestido, salud, protección y cariño, por lo cual en este artículo se comenta en torno al tema y se expone la experiencia traumática vivida por 2 menores, lo que en nuestro estudio demuestra en una muestra de dos años la alta incidencia de maltrato en menores de 5 a 9 años de edad.

Sin embargo, Tovar A y colaboradores realizaron una investigación con el objetivo de conocer la percepción que tienen las niñas y los niños acerca del maltrato infantil, demostrando de la misma manera la alta incidencia de patología postraumática, la afectación psicológica en los niños y su adaptación en el sistema social y la reinserción educativa.

Sin embargo, Pinto C; Moraga C; Henríquez D. en su estudio de experiencias de polivictimización como predictores de síntomas postraumáticos realizada en el 2018, han demostrado al igual que nuestro estudio que mediante la interacción entre paciente y valoración psicológica se determinan los efectos traumáticos y postraumáticos, que han predicho significativamente los síntomas iniciales en los niños maltratados estudiados.

Por tal razón, en Ecuador en el año 2010 demuestra que 4 de cada 10 niños, niñas y adolescentes entre 5 y 17 años recibieron un trato violento de sus padres por cometer un error o no obedecer, según datos del Observatorio de los Derechos de la Niñez y Adolescencia, ${ }^{2}$ lo cual al comprarlo con nuestro estudio, demuestra la afectación a 347 pacientes, es decir una alta incidencia, con sintomatologías muy similares en los diferentes tipos de maltrato físico, psicológico y sexual.

\section{Conclusiones}

Se identificó los síntomas iniciales de niños con maltrato infantil en el aspecto físico, psicológico y sexual en los pacientes atendidos en Sala de Primera Acogida del Hospital General Docente Ambato en el año 2017, para la obtención de un diagnóstico presuntivo. La sintomatología va reportada desde el aspecto médico-clínico como a su vez del ámbito psicológico relacionándose con los síntomas conductuales en las que se identifican al miedo, la depresión, los trastornos del sueño y de la alimentación en los dos grupos etarios tanto de 5 a 9 años como de 10 a 14 años, técnicamente podemos darnos cuenta que captados a corta edad puede ayudarnos a prevenir enfermedades y eventos postraumáticos radicales en el desarrollo cotidiano. La sintomatología en menores de edad es posible que no tengan un factor desencadenante fijo, ya que puede simplemente ser circunstancial visto por diferentes aspectos entre ellos económicos, culturales, sociales, emocionales y biológicas, cada una con las características propias de su entorno, que al resultar una inquietud nos permitieron empoderarnos para poder realizar este estudio. Dentro del estudio en la sintomatología psicológica nos hemos dado cuenta que la mayoría de los niños presentan miedo y este se puede expresar en el temor por lo cual callan su situación actual, y dificultando la captación de indicadores de maltrato, pero pese a este inconveniente el paciente requiere todo el apoyo del personal de salud y de una exploración clínica y psicológica minuciosa, a la cual nosotros la podemos llamar como tamizaje universal. Concretamente, las investigaciones realizadas en los últimos años han encontrado que la sustitución de estrategias de afrontamiento de evitación por estrategias de aproximación, la eliminación de las atribuciones de autoinculpación e inculpación a la familia y de los sentimientos relacionados con las cuatro dinámicas traumatogénicas y la promoción de un estilo de apego seguro resultarían beneficiosos a la hora de prevenir futuros problemas causados por la experiencia de abuso. 


\section{Recomendaciones}

Capacitaciones continúas sobre el manejo adecuado del protocolo de atención de la norma técnica de violencia de género, y su tamizaje a todo el personal rotativo de cada una de las instituciones públicas y privadas de la Provincia, que permitan el seguimiento adecuado y oportuno a los niños que han sido víctimas de cualquier tipo de violencia y evitar así efectos postraumáticos letales, no solo para el menor de edad sino también en el entorno socio-familiar.

Realización de un buen control médico y psicológico en el periodo de tiempo adecuado, siendo lo óptimo para un estricta valoración evolutiva al mes, a los tres meses y a los 6 meses, en busca de alteraciones clínicas como psicológicas, o facultadas mediante la valoración física como la toma oportuna de exámenes de laboratorio.

\section{Conflicto de intereses}

Ninguno declarado por los autores.

\section{Financiación}

Autofinanciado.

\section{Agradecimientos}

Ninguno declarado por los autores.

\section{Referencias}

1. SENPLADES. Toda Una Vida: Plan Nacional de Desarrollo 2017-2021 Quito: Secretaría Nacional de Planificación y Desarrollo, Senplades.; 2017

2. deas Infantil. Aldeas Infantil SOS Ecuador. [Online].; 2017 . Available from: https://www.aldeasinfantiles.org.ec/informate/ultimas-noticias/violenciaintrafamiliar-datos-y-estadisticas-en-ec

3. inheiro P. (2018) Informe Muldial sobre la violencia contra los niños y niñas, Unicef, Sao Paulo-Brasil. (Editada el 6 de mayo 2018 ; Citado el 10 de enero del 2019). Available from: https://www.unicef.org/lac/Informe_Mundial_Sobre_Violencia_1(1).pdf

4. Arévalo Barea AR. Abuso Infantil. Rev. Méd. La Paz. 2016 Mayo; 22(2

5. Guerra C, Chamarrita F, Sintomatología en víctimas de abuso sexual: Son importantes las características objetivas del abuso. Revista de Psicología de Chile, 2015, 24(2), 1-19\}

6. Tovar A, Almeraya S, Guajardo L, Borja M (2016) Child abuse from the voice of children. Revista Mexicana de Ciencias Agrícolas; 7(1):195-207.

7. Pinto C; Moraga C; Henríquez D. Experiencias de polivictimización como predictoras de síntomas postraumáticos en una muestra de adolescentes chilenos, Interciencia, vol. 43, núm. 5, 2018, pp. 329-335, Asociación interciencia. Caracas, Venezuela

8. Flórez M y González A (2011), Caracterizar la violencia intrafamiliar, infantil y sexual en Bogotá D.C-Colombia, durante el año 2011. Teoría y Praxis Investigativa. Bogotá, Colombia; 8(1):74-91.

9. Rizo Martínez Lucía Ester, Guevara Pérez Miguel Ángel, Hernández González Marisela, Sánchez Sosa Juan José. A preliminary study of the prevalence of post-traumatic stress disorder, depression and anxiety symptoms in female adolescents maltreatment victims in Mexico. Salud Ment [revista en la Internet]. 2018, 41(3): 139-144. Disponible en: http://www.scielo.org.mx/scielo.php?script=sci_arttext\&pid=S0185-33252018000300139\&Ing=es. http://dx.doi.org/10.17711/sm.0185-3325.2018.018

10. Cantón D, Cortés M. Consecuencias del abuso sexual infantil: una revisión de las variables intervinientes. Anal. Psicol. [Internet]. 2015 Mayo [citado 2019 Feb 17] ; 31( 2 ): 607-614. Disponible en: http://scielo.isciii.es/scielo.php?script=sci_arttext\&pid=S0212-97282015000200024\&Ing=es. http://dx.doi.org/10.6018/analesps.31.2.180771. 